\title{
The Improving Rural Cancer Outcomes Trial: a cluster-randomised controlled trial of a complex intervention to reduce time to diagnosis in rural cancer patients in Western Australia
}

Jon D Emery, Victoria Gray, Fiona M Walter, Shelley Cheetham, Emma J Croager, Terry Slevin, Christobel Saunders, Timothy Threlfall, Kirsten Auret, Anna K Nowak, Elizabeth Geelhoed, Max Bulsara and C D'Arcy J Holman

Correction to: British Journal of Cancer (2017) 117, 1459-1469; doi:10.1038/bjc.2017.310; published online 19 September 2017

There was an error in Table 3 of this manuscript and some of the 25 th and 75 th percentile values were transposed. The corrected Table 3 is shown here.
In addition, there was an error in Table $4 \mathrm{c}$ for the community control group with prostate cancer. The 25 th and 75 th percentiles were transposed. The corrected Table $4 \mathrm{c}$ is shown here.References

Table 3. Total diagnostic interval by trial group, factorial design and tumour type

\begin{tabular}{|c|c|c|c|c|c|c|c|c|c|c|c|c|c|c|c|c|c|c|c|c|c|c|c|c|c|c|}
\hline \multirow{2}{*}{$\begin{array}{l}\text { Trial } \\
\text { group }\end{array}$} & \multicolumn{6}{|c|}{ Breast } & \multicolumn{5}{|c|}{ Colorectal } & \multicolumn{5}{|c|}{ Lung } & \multicolumn{5}{|c|}{ Prostate } & \multicolumn{5}{|c|}{ Total } \\
\hline & $n$ & $\underset{\substack{\text { Mean } \\
\mathrm{Ln}^{*}}}{ }$ & Mean & Med'n & 25th & 75th & $n$ & $\begin{array}{c}\text { Mean } \\
\mathrm{Ln}^{*}\end{array}$ & Med'n & 25th & 75th & $n$ & $\begin{array}{c}\text { Mean } \\
\operatorname{Ln}^{*}\end{array}$ & Med'n & 25th & 75th & $n$ & $\begin{array}{c}\text { Mean } \\
\operatorname{Ln}^{*}\end{array}$ & Med'n & 25th & 75th & $n$ & $\begin{array}{c}\text { Mean } \\
\mathrm{Ln}^{*}\end{array}$ & Med'n & 25th & 75th \\
\hline $\mathrm{Cl}$ & 143 & 3.80 & 97.5 & 33 & 21 & 115 & 103 & 4.44 & 177.7 & 53 & 107 & 48 & 4.64 & 190.6 & 46.3 & 114.5 & 188 & 4.98 & 212.1 & 86 & 161.5 & 482 & 4.48 & 168.6 & 36 & 107.5 \\
\hline cc & 275 & 3.67 & 82.5 & 34 & 18 & 97 & 146 & 4.70 & 195.3 & 53 & 133 & 65 & 4.58 & 157.4 & 53.5 & 114 & 346 & 4.82 & 186.8 & 66.8 & 123.5 & 832 & 4.40 & 151.5 & 35 & 92 \\
\hline GPI & 176 & 3.77 & 83.0 & 34.5 & 19.3 & 100.8 & 118 & 4.54 & 185.3 & 43 & 124 & 55 & 4.66 & 180.7 & 55 & 115 & 199 & 4.88 & 192.9 & 74 & 139 & 548 & 4.43 & 154.7 & 35 & 97 \\
\hline GPC & 196 & 3.69 & 91.8 & 33 & 18 & 91.5 & 94 & 4.57 & 185.3 & 57.3 & 122 & 45 & 4.64 & 170.4 & 49 & 125 & 253 & 4.93 & 203.1 & 76.5 & 148 & 588 & 4.42 & 160.7 & 34.25 & 96.5 \\
\hline $\begin{array}{l}\mathrm{Cl} \text { and } \\
\mathrm{GPI}\end{array}$ & 56 & 3.83 & 89.3 & 37 & 19.3 & 121 & 49 & 4.41 & 170.3 & 43 & 107 & 26 & 4.40 & 162.4 & 36.3 & 103.5 & 72 & 4.95 & 200.4 & 86.3 & 143 & 203 & 4.44 & 157.6 & 36 & 100 \\
\hline $\begin{array}{l}\mathrm{CC} \text { and } \\
\mathrm{GPI}\end{array}$ & 120 & 3.74 & 80.1 & 34 & 19.3 & 97.8 & 69 & 4.64 & 195.9 & 42.5 & 128 & 29 & 4.89 & 197.1 & 71.5 & 119 & 127 & 4.83 & 188.6 & 69 & 129 & 345 & 4.42 & 153.0 & 34.5 & 92 \\
\hline $\begin{array}{l}\mathrm{Cl} \text { and } \\
\mathrm{GPC}\end{array}$ & 68 & 3.71 & 99.1 & 31 & 21.3 & 88.8 & 38 & 4.34 & 179.7 & 62 & 98 & 13 & 5.06 & 263.2 & 56.5 & 173 & 86 & 5.01 & 222.9 & 79.8 & 163.5 & 205 & 4.46 & 176.4 & 33.5 & 103 \\
\hline $\begin{array}{l}\mathrm{CC} \text { and } \\
\text { GPC }\end{array}$ & 128 & 3.63 & 87.9 & 33.5 & 17.3 & 92 & 56 & 4.73 & 189.2 & 55.8 & 134 & 32 & 4.47 & 132.7 & 42.8 & 11.05 & 167 & 4.88 & 193.0 & 73 & 132 & 383 & 4.41 & 152.3 & 35 & 95 \\
\hline
\end{tabular}

Abbreviations: $\mathrm{CC}=$ community control; $\mathrm{Cl}=$ community intervention; $\mathrm{GPC}=\mathrm{GP}$ con; $\mathrm{GPI}=\mathrm{GP}$ intervention.

${ }^{a}$ Natural log of mean. All other values in days. 
Table 4c. Specialist interval by trial group, by factorial design and tumour type

\begin{tabular}{|c|c|c|c|c|c|c|c|c|c|c|c|c|c|c|c|c|c|c|c|c|c|c|c|c|c|c|c|c|c|c|}
\hline \multirow{2}{*}{\begin{tabular}{|l|} 
Trial \\
group
\end{tabular}} & \multicolumn{6}{|c|}{ Breast } & \multicolumn{6}{|c|}{ Colorectal } & \multicolumn{6}{|c|}{ Lung } & \multicolumn{6}{|c|}{ Prostate } & \multicolumn{6}{|c|}{ Total } \\
\hline & $n$ & $\begin{array}{l}\text { Mean } \\
\mathrm{Ln}^{*}\end{array}$ & Mean & Med'n & 25th & 75th & ${ }^{\mathrm{n}}$ & \begin{tabular}{|c|} 
Mean \\
$\mathrm{Ln}^{*}$ \\
\end{tabular} & Mean & Med'n & 25th & 75th & $\mathrm{n}$ & $\begin{array}{c}\text { Mean } \\
\mathrm{Ln}^{*}\end{array}$ & Mean & Med'n & 25th & 75th & $n$ & \begin{tabular}{|c|} 
Mean \\
$\mathrm{Ln}^{*}$ \\
\end{tabular} & Mean & Med'n & 25th & 75th & $\mathrm{n}$ & \begin{tabular}{|c|} 
Mean \\
$\mathrm{Ln}^{*}$
\end{tabular} & Mean & Med'n & 25th & 75th \\
\hline $\mathrm{Cl}$ & 86 & 1.91 & 20.0 & 13 & 5.8 & 23 & 77 & 3.40 & 54.1 & 36 & 14 & 71 & 27 & 2.53 & 40.6 & 16 & 21 & 72 & 165 & 4.36 & 114.2 & 83 & 165 & 228 & 355 & 3.42 & 72.6 & 39 & 15 & 91 \\
\hline $\mathrm{cc}$ & 146 & 1.70 & 22.3 & 11.5 & 4 & 23.3 & 133 & 3.48 & 71.6 & 39 & 21 & 72 & 47 & 2.80 & 28.3 & 16 & 8 & 36 & 330 & 4.14 & 94.4 & 63 & 63 & 330 & 656 & \begin{tabular}{|l|}
3.37 \\
\end{tabular} & 69.0 & 41 & 16 & 78.8 \\
\hline GPI & 95 & 2.04 & 23.9 & 13 & 6 & 29 & 100 & 3.61 & 76.4 & 41 & 23 & 93 & 38 & 2.64 & $\mid$\begin{tabular}{|l}
$\mid$ \\
$\mid$
\end{tabular} & 14.5 & 6.75 & 26.5 & 183 & 4.29 & \begin{tabular}{|l|}
106.8 \\
\end{tabular} & 73 & 38 & 140 & 416 & 3.36 & 73.1 & 39 & 16 & 93 \\
\hline GPC & 113 & 1.49 & 17.3 & 10 & 5 & 21.8 & 8 & 3.36 & 60.3 & 36 & 15.5 & 58.5 & 31 & 2.84 & \begin{tabular}{|l|}
44.2 \\
\end{tabular} & 19 & 11 & 36 & 240 & 4.17 & 98.3 & 68 & 38 & 119 & 467 & \begin{tabular}{|l|}
3.29 \\
\end{tabular} & 68.4 & 40.5 & 14 & 77 \\
\hline $\begin{array}{l}\mathrm{Cl} \text { and } \\
\mathrm{GPI}\end{array}$ & 32 & 1.20 & 19.8 & 15.5 & 5 & 27.5 & 35 & 3.60 & 62.4 & 42 & 21 & 94 & 17 & 2.56 & $\mid 17.2$ & 16 & 10 & 24 & 62 & 4.34 & 109.8 & 91 & 37.75 & 140.8 & 146 & 3.44 & 67.9 & 37.5 & 16 & 93.3 \\
\hline $\begin{array}{l}\mathrm{CC} \text { and } \\
\mathrm{GPI}\end{array}$ & 63 & 2.06 & 25.9 & 13 & 6 & 29 & 6 & \begin{tabular}{|l}
3.62 \\
\end{tabular} & 83.9 & 40 & 23 & 92.5 & 21 & 2.70 & 31.9 & 14 & 6 & 40.5 & 121 & 4.27 & 105.2 & 71 & 38 & 140.5 & 270 & 3.48 & 75.9 & 41 & 15.5 & 92 \\
\hline $\begin{array}{l}\mathrm{Cl} \text { and } \\
\text { GPC }\end{array}$ & 44 & 1.56 & 15.7 & 11 & 5.3 & 19.5 & 31 & \begin{tabular}{|l}
3.27 \\
\end{tabular} & 47.0 & 28 & 13 & 57 & 7 & 2.10 & 102 & 12 & 5 & 28 & 78 & 4.33 & 115.2 & 76.5 & 48 & 138.3 & 160 & \begin{tabular}{|l|} 
\\
\end{tabular} & 74.0 & 39 & 13.3 & 84 \\
\hline $\begin{array}{l}\mathrm{CC} \text { and } \\
\text { GPC }\end{array}$ & 69 & 1.44 & 18.3 & 10 & 4 & 22 & 8 & 3.40 & 68.3 & 41 & 17 & 60 & 24 & 3.06 & 27.4 & 19 & 11.75 & 44.25 & 162 & 4.10 & 90.2 & 62 & 35 & 111 & 307 & 3.30 & 65.4 & 41 & 16 & 73.8 \\
\hline
\end{tabular}

Abbreviations: $\mathrm{CC}=$ community control; $\mathrm{Cl}=$ community intervention; $\mathrm{GPC}=\mathrm{GP}$ control; $\mathrm{GPI}=\mathrm{GP}$ intervention.

${ }^{a}$ Natural log of mean. All other values in days.

(c) (i) (2)(2) This work is licensed under the Creative Commons Cay Attribution-Non-Commercial-Share Alike 4.0 International License. To view a copy of this license, visit http:// creativecommons.org/licenses/by-nc-sa/4.0/

(C) The Author(s) named above 2018 